\title{
Vibration control systems
}

Hamid Reza Karimi

Faculty of Technology and Science, University of Agder, Grimstad,

Norway

E-mail address: hamid.r.karimi@uia.no

Huijun Gao

Research Institute of Intelligent Control and Systems, Harbin Institute of

Technology, P.O. Box 3015, Harbin 150080, PR China

E-mail address: hjgao@hit.edu.cn
James Lam

Department of Mechanical Engineering, The University of Hong Kong, Hong Kong

E-mail address: james.lam@hku.hk

Haiping Du

School of Electrical, Computer and Telecommunications Engineering, University of Wollongong, Australia

E-mail address: hdu@uow.edu.au
Vibration is a phenomenon that affects systems such as bridges, buildings, towers, vehicles and aircraft. The protection of these systems against the harmful effects of vibration has become a major field of research in recent years. This special issue has a strong impact in many real-world systems, like vehicles and active suspension systems, buildings, wind turbine systems, and motion systems.

The overall aims of this special issue are to promote discussion among researchers actively working in the vibration control systems and to provide an up-to-date overview of the research directions and advance control strategies in the field. This special issue contains eleven papers, the contents of which are summarized as follows.

Liu et al. present a novel technique to identify a spatial linear parameter varying model using a local approach, such that the input/output behaviour from any actuator to sensor on a structure is parametrized. Experimental results on an actuated beam demonstrate the performance of the proposed approach.

Wang et al. develop a robust data-driven fault detection scheme for wind turbines. The fault detection scheme contains two steps, i.e. residual generation, and residual evaluation including threshold computation and decision making. In the first step, a robust residual vector instead of a single residual signal is generated under a given performance index and an optimization criterion. In the second step, a proper evaluation approach as well as a suitable decision logic is given to make a correct final decision.

Ronde et al. introduce a novel feedforward design method, called spatial feedforward, for over-actuated flexible motion systems. Also, conditions for the existence of spatial feedforward are explicitly derived. Furthermore, this method has been experimentally validated on an industrial prototype lightweight motion system.

Sun et al. present a constrained adaptive backstepping control scheme for active suspensions to achieve the multi-objective control, such that the resulting closed-loop systems can improve ride comfort and at the same time satisfy the performance constraints in the presence of parametric uncertainties. Also, a design example is shown to illustrate the effectiveness of the proposed control law, where different initial state values are considered in order to verify the proposed approach in detail.

Su et al. design a fuzzy controller for the electromagnetic suspension (EMS) system of maglev train. This controller is designed such that, in presence of energy bounded disturbance, the position offset of maglev train is within an allowable scale. Consequently the comfort level of high speed maglev train will be greatly improved. Finally, simulation results are provided to verify the feasibility and effectiveness of our controller design method.
Palacios-Quinonero et al. present the problem of local velocityfeedback energy-to-peak controller design for the seismic protection of a two-building system. This approach comprises conceptual, computational, and methodological aspects. To assess the effectiveness of the proposed controller, numerical simulations are conducted with positive results.

Cazzulani et al. propose exploiting the measurements of optical strain gauges, such as Fiber Bragg Grating (FBG) sensors as feedback for active vibration control applications. Experimental tests confirm that smart structures with embedded FBG sensors can be profitably designed to suppress vibrations.

Zhang et al. study the problem of robust finite frequency $H_{\infty}$ passive fault-tolerant static-output feedback controller design and apply it to an active control problem of a structural system under an earthquake excitation. Simulations and comparisons have shown that the designed fault-tolerant controller can significantly attenuate the vibration from the ground and protect the structural system even actuator faults occur.

$\mathrm{Gu}$ et al. propose a combined control scheme for high-bandwidth control of piezoceramic stack actuators (PSAs). Comparative experiments on a PSA-actuated stage are conducted in real time to verify the effectiveness of the proposed control scheme.

Zhang et al. investigate a dual-type (strain gauge and displacement transducer) sensor placement method with the aim of the possibly best reconstruction of multi-scale structural responses. An experimental work is then designed with the simply supported overhanging beam. Finally, the results show that the proposed method is feasible and efficient.

Heertjes and Verstappen propose a sliding mode controller with saturated integrator to improve the tracking problem in highprecision motion systems. The proposed controller with optimized parameters is implemented on an industrial wafer scanner to improve throughput during the wafer stage (chuck) exchange.

We would like to thank all the authors for contributing submissions to the special issue. Also, we would like to acknowledge all the anonymous reviewers for the voluntary work. Finally, we really appreciate the editor-in-chief of Mechatronics, Prof. Maarten Steinbuch for his great support to our special issue. 